\title{
Embeddings of non-simply-connected 4-manifolds in 7-space. II. On the smooth classification
}

\author{
D. Crowley*and A. Skopenkov ${ }^{\dagger}$
}

\begin{abstract}
We work in the smooth category. Let $N$ be a closed connected orientable 4-manifold with torsion free $H_{1}$, where $H_{q}:=H_{q}(N ; \mathbb{Z})$. Our main result is a readily calculable classification of embeddings $N \rightarrow \mathbb{R}^{7}$ up to isotopy, with an indeterminancy. Such a classification was only known before for $H_{1}=0$ by our earlier work from 2008. Our classification is complete when $H_{2}=0$ or when the signature of $N$ is divisible neither by 64 nor by 9 .

The group of knots $S^{4} \rightarrow \mathbb{R}^{7}$ acts on the set of embeddings $N \rightarrow \mathbb{R}^{7}$ up to isotopy by embedded connected sum. In Part I we classified the quotient of this action. The main novelty of this paper is the description of this action for $H_{1} \neq 0$, with an indeterminancy.

Besides the invariants of Part I, detecting the action of knots involves a refinement of the Kreck invariant from our work of 2008.

For $N=S^{1} \times S^{3}$ we give a geometrically defined $1-1$ correspondence between the set of isotopy classes of embeddings and a certain explicitly defined quotient of the set $\mathbb{Z} \oplus \mathbb{Z} \oplus \mathbb{Z}_{12}$.
\end{abstract}

\section{Contents}

1 Overview and main results 2

2 Definition of the invariants $\quad 5$

3 Proof of Corollaries 1.4 and 1.8.bc $r$

4 Proof of Lemma 2.1.b 10

5 Proof of Lemma 2.1.ac $r$

6 Idea of the proof of Lemma 2.1 15

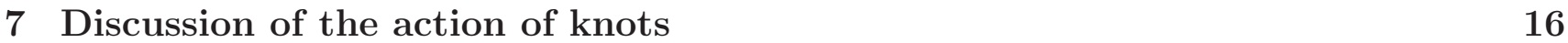

*The University of Melbourne, Australia. Email: dcrowley@unimelb.edu.au. Web: www.dcrowley.net .

$\dagger$ Moscow Institute of Physics and Technology, and Independent University of Moscow, Russia. Email: skopenko@mccme.ru. Web: https://users.mccme.ru/skopenko/. Supported in part by the Russian Foundation for Basic Research Grants No. 15-01-06302 and 19-01-00169, by Simons-IUM Fellowship and by the D. Zimin Dynasty Foundation. 


\section{Overview and main results}

We consider smooth manifolds, embeddings and isotopies. For an $n$-manifold $P$ denote by $E^{m}(P)$ the set of isotopy classes of embeddings $P \rightarrow S^{m}$. The group $E^{m}\left(S^{n}\right)$ acts on $E^{m}(P)$ by embedded connected sum [CS16, §1.1], [Sk16c, §4]. Denote this action by \# and its quotient by $E_{\#}^{m}(P)$.

Remark 1.1 (The action of knots in general). If the quotient $E_{\#}^{m}(P)$ is known for a closed $n$-manifold $P$, the description of $E^{m}(P)$ is reduced to the determination of the orbits of the embedded connected sum action of $E^{m}\left(S^{n}\right)$ on $E^{m}(P)$. For a general closed $n$-manifold $P$ describing the action by a non-zero group of knots $E^{m}\left(S^{n}\right)$ on $E^{m}(P)$ is a non-trivial task. For the cases when the quotient $E_{\#}^{7}(N)$ coincides with the set of PL embeddings up to PL isotopy, the quotient has been known since 1960s [Sk16e, Sk16f, Sk16t]. However, until recently no description of the action (or, equivalently, no classification of $E^{m}(P)$ ) was known for $E^{m}\left(S^{n}\right) \neq 0$ and $P$ not a disjoint union of homology spheres. For recent results see [Sk08', Sk10, CS11]. On the other hand, the description of the action in [CRS07, Sk11, CRS11, Sk15] is not hard, the hard part of the cases considered there is rather the description of the quotient $E_{\#}^{m}(P)$.

There are non-isotopic embeddings $g_{1}, g_{2}: S^{2} \rightarrow S^{4}$ and an embedding $f: \mathbb{R} P^{2} \rightarrow S^{4}$ such that $\left[f \# g_{1}\right] \neq\left[f \# g_{2}\right]$ [Vi73]; i.e. the action of the monoid $E^{4}\left(S^{2}\right)$ on $E^{4}\left(\mathbb{R} P^{2}\right)$ is not free.

Various authors have studied the analogous connected sum action of the group of homotopy $n$-spheres on the set of smooth $n$-manifolds homeomorphic to given manifold; see for example [Le70, Sc73, Wi74] and references there.

More motivation and background for this paper may be found in [Sk16c, Sk16f] and Part I [CS16, §1]. In this paper $N$ is a closed connected orientable 4-manifold and $H_{q}:=H_{q}(N ; \mathbb{Z})$. We present a readily calculable classification (in the sense of [Sk16c, Remark 1.2]) of $E^{7}(N)$ when $H_{1}$ is torsion free (up to an indeterminancy in certain cases). See Theorems 1.2, 1.6 and Corollaries 1.5, 1.8 below. Our classification is complete when $H_{2}=0$ (see Theorem 1.6 and Corollary 1.8.b) or when the signature of $N$ is divisible neither by 64 nor by 9 (see Theorem 1.6 and Corollary 1.5). The classification requires finding a complete set of invariants and constructing embeddings realizing particular values of these invariants. The invariants we use are described in [CS16, Lemma 1.3, $\S 2.2, \S 2.3$ ] and $\S 2$. An overview of the proof of their completeness is given in [CS16, $\S 1.4$ ] and in Remark 7.4 below (using definitions recalled at the beginning of $\S 2$ ).

The action of $E^{7}\left(S^{4}\right) \cong \mathbb{Z}_{12}$ on $E^{7}(N)$ was investigated in [Sk10] and determined when $H_{1}=0$ in [CS11], which also classified $E^{7}(N)$ in this case. In [CS16] we described the quotient $E_{\#}^{7}(N)$ when $H_{1}=0$. Thus the main novelty of this paper is the description of this action for $H_{1} \neq 0$.

Denote by $q_{\#}: E^{7}(N) \rightarrow E_{\#}^{7}(N)$ the quotient map.

Let us state our main result for $N=S^{1} \times S^{3}$. For this identify $E^{7}\left(S^{4}\right)$ and $\mathbb{Z}_{12}$ by the isomorphism $\eta$ of [CS11] (recalled in a more general situation in §2) and consider the following diagram (where the left triangle is not commutative):

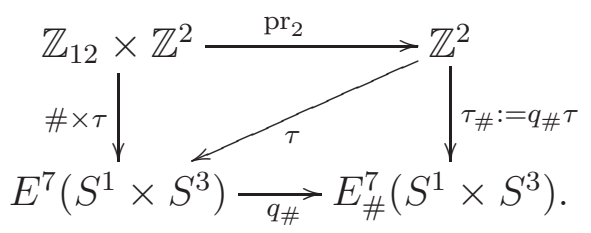

The map $\tau$ is defined in [CS16, §1.2]. We define the map \# $\times \tau$ by $(\# \times \tau)(a, l, b):=a \# \tau(l, b)$.

Theorem 1.2. The map $\# \times \tau: \mathbb{Z}_{12} \times \mathbb{Z}^{2} \rightarrow E^{7}\left(S^{1} \times S^{3}\right)$ is a surjection such that

(a) for different pairs $l, b$ the sets $P_{l, b}:=(\# \times \tau)\left(\mathbb{Z}_{12} \times(l, b)\right)$ either are disjoint or coincide;

$$
P_{l, b}=P_{l^{\prime}, b^{\prime}} \quad \Leftrightarrow \quad\left(l=l^{\prime} \quad \text { and } \quad b \equiv b^{\prime} \bmod 2 l\right) ;
$$




$$
\text { (c) } \quad\left|P_{l, b}\right|= \begin{cases}12 & l \neq 0 \\ 2 \operatorname{gcd}(b, 6) & l=0 .\end{cases}
$$

In Theorem 1.2 the surjectivity of $\tau$ and Parts (a) and (b) follow from [CS16, Theorem 1.1]. The new part of Theorem 1.2 is (c); this part follows from Corollary 1.8.b below (because for $l \neq 0$ the group coker $\bar{l}$ is finite, so $\operatorname{div} b=0)$. Cf. Addendum 7.3.

Example 1.3. There is an embedding $f: S^{1} \times S^{3} \rightarrow S^{7}$ with $f(N) \subset S^{6}$ and a pair of non-isotopic embeddings $g_{1}, g_{2}: S^{4} \rightarrow S^{7}$ such that $f \# g_{1}$ and $f \# g_{2}$ are isotopic.

This example follows because $\left|P_{0,1}\right|=2$ by Theorem 1.2 and there is a representative of $\tau(0,1)$ whose image is in $S^{6} \subset S^{7}$ [CS16, Lemma 2.18]. Example 1.3 shows the necessity of the simple-connectivity assumption in the following result (which is [Sk10, The Effectiveness Theorem 1.2]):

If $f: N \rightarrow S^{7}$ is an embedding of a spin simply-connected closed 4-manifold $N, f(N) \subset S^{6}$ and embeddings $g_{1}, g_{2}: S^{4} \rightarrow S^{7}$ are not isotopic, then $f \# g_{1}$ and $f \# g_{2}$ are not isotopic.

Before stating our main result for the general case in Theorem 1.6 below we state the following corollaries of it.

Corollary 1.4 (of Theorem 1.6.c; proved in $\S 3$ ). Let $N$ be a closed connected orientable 4manifold with torsion free $H_{1}$. Then the following statements are equivalent:

(i) for every embedding $f: N \rightarrow S^{7}$ and non-isotopic embeddings $g_{1}, g_{2}: S^{4} \rightarrow S^{7}$ the embeddings $f \# g_{1}$ and $f \# g_{2}$ are not isotopic (i.e. the action \# of $E^{7}\left(S^{4}\right)$ on $E^{7}(N)$ is free);

(ii) $N$ is an integral homology 4-sphere.

The Boéchat-Haefliger invariant $\varkappa_{\#}$ is defined in [CS16, $\S 2.2$, and we denote $\varkappa_{\#} \varkappa_{\#} q_{\#}$ :

$$
E^{7}(N) \stackrel{q_{\#}}{\longrightarrow} E_{\#}^{7}(N) \stackrel{\varkappa_{\#}}{\longrightarrow} H_{2}^{D I F F}:=\left\{u \in H_{2} \mid \rho_{2} u=w_{2}^{*}(N), u \cap_{N} u=\sigma(N)\right\} \subset H_{2}
$$

Corollary 1.5 (of Theorem 1.6.c). Let $N$ be a closed connected orientable 4-manifold with torsion free $H_{1}$ and $f: N \rightarrow S^{7}$ an embedding.

(a) If $\varkappa(f)$ is neither divisible by 4 nor by 3 , then for every embedding $g: S^{4} \rightarrow S^{7}$ the embeddings $f \# g$ and $f$ are isotopic.

(b) If $\varkappa(f)$ is divisible by 4 but neither by 8 nor by 3, then there is a non-trivial embedding $g_{1}: S^{4} \rightarrow S^{7}$ such that for every embedding $g: S^{4} \rightarrow S^{7}$ the embedding $f \# g$ is isotopic to either $f$ or $f \# g_{1}$.

Corollary 1.5 follows from Corollary 1.8.bc (or from Theorem 1.6.c and Addendum 1.7 because $4 \mathbb{Z}_{\operatorname{gcd}(\varkappa(f), 24)}=0$ under the assumptions of Corollary 1.5). The assumption of Corollary 1.5.a is automatically satisfied when the signature of $N$ is divisible neither by 16 nor by 9 .

Denote

$$
\widehat{n}:=\operatorname{gcd}(n, 24) .
$$

If $H_{1}=0$, then $\varkappa_{\#}$ is $1-1$ and $\varkappa=\varkappa_{\#} q_{\#}$ is surjective. For each $u \in H_{2}^{D I F F}$ we have $\left|\varkappa^{-1}(u)\right|=$ $\widehat{u} / \operatorname{gcd}(u, 2)$. Here the first sentence is easily deduced from [BH70], see [CS16, Remark 2.20.e]. The second sentence is proved in [CS11].

Our second main result is a generalization of the above statement to non-simply-connected 4-manifolds. We use the convention on coefficients and the notation for characteristic classes and intersections in manifolds from [CS16, §1.2].

Definition of div, $B\left(H_{3}\right), \bar{l}$, a symmetric pair, $K_{u, l}, C_{u, l}$ and $\cap_{d}$. For an element $u$ of a free abelian group denote by $\operatorname{div} u$ the divisibility of $u$, i.e. $\operatorname{div} 0=0$ and $\operatorname{div} u$ is the largest 
integer which divides $u$ for $u \neq 0$. For an element $u$ of an abelian group $G$ denote by $\operatorname{div} u$ the divisibility of $[u] \in G / \operatorname{Tors}(G)$.

Denote by $B\left(H_{3}\right)$ the space of bilinear forms $H_{3} \times H_{3} \rightarrow \mathbb{Z}$. For $l \in B\left(H_{3}\right)$ denote by $\bar{l}: H_{3} \rightarrow H_{1}$ the adjoint homomorphism uniquely defined by the property $l(x, y)=x \cap_{N} \bar{l} y$. A pair $(u, l) \in H_{2} \times B\left(H_{3}\right)$ is called symmetric if

$$
l(y, x)=l(x, y)+u \cap_{N} x \cap_{N} y \quad \text { for all } \quad x, y \in H_{3} .
$$

For $u \in H_{2}, l \in B\left(H_{3}\right)$ and $d:=\operatorname{div} u \in \mathbb{Z}$ denote

$$
K_{u, l}:=\operatorname{ker}\left(2 \rho_{d} \bar{l}\right) \subset H_{3} \quad \text { and } \quad C_{u, l}:=\operatorname{coker}\left(2 \rho_{d} \bar{l}\right) .
$$

If the pair $(u, l)$ is symmetric, then a bilinear map

$$
\cap_{d}: C_{u, l} \times K_{u, l} \rightarrow \mathbb{Z}_{d} \quad \text { is well-defined by } \quad[x] \cap_{d} y:=x \cap_{N} y \cdot{ }^{1}
$$

The maps $\varkappa, \lambda, \beta_{u, l}$, and $\eta_{u, l, b}, \theta_{u, l, b}$ of Theorem 1.6 below are defined in $\S 2$. The definitions of $\varkappa, \lambda, \beta_{u, l}$ are recalled from [CS16, $22.2, \S 2.3$ ] and the definitions of $\eta_{u, l, b}$ and $\theta_{u, l, b}$ are new.

Theorem 1.6. Let $N$ be a closed connected orientable 4-manifold with torsion free $H_{1}$.

(a) The product

$$
\varkappa \times \lambda: E^{7}(N) \rightarrow H_{2}^{D I F F} \times B\left(H_{3}\right)
$$

has non-empty image consisting of symmetric pairs.

(b) For every $(u, l) \in \operatorname{im}(\varkappa \times \lambda)$ denote $d:=\operatorname{div} u$. Every map

$$
\beta_{u, l}:(\varkappa \times \lambda)^{-1}(u, l) \rightarrow C_{u, l}
$$

is surjective (see the remark immediately below the Theorem).

(c) For every $b \in C_{u, l}$ every map

$$
\eta_{u, l, b}: \beta_{u, l}^{-1}(b) \rightarrow \frac{\mathbb{Z}_{\widehat{d}}}{\operatorname{im} \theta_{u, l, b}}
$$

is an injection whose image consists of all even elements (see the remark immediately below the Theorem). Moreover, the map

$$
\theta_{u, l, b}: K_{u, l} \rightarrow 4 \mathbb{Z}_{\widehat{d}}
$$

is a homomorphism and

$$
\begin{aligned}
& \quad \theta_{u, l, b}(y)-\theta_{u, l, b^{\prime}}(y)=4 \rho_{\widehat{d}}\left(b-b^{\prime}\right) \cap_{d} y \quad \text { for every } \quad b, b^{\prime} \in C_{u, l} \quad \text { and } \quad y \in K_{u, l} \text {. } \\
& \text { (d) }\left|\beta_{u, l}^{-1}(b)\right|=\frac{\widehat{u}}{\operatorname{gcd}(u, 2) \cdot\left|\operatorname{im} \theta_{u, l, b}\right|} .
\end{aligned}
$$

We call geometrically defined maps invariants. In particular, the maps $\lambda$ and $\varkappa$ are invariants.

Remark on relative invariants. The maps $\beta_{u, l}$ and $\eta_{u, l, b}$ are relative invariants. For $\eta_{u, l, b}$ this means that for $\left[f_{0}\right],\left[f_{1}\right] \in \beta_{u, l}^{-1}(b)$ there is an invariant $\left(\left[f_{0}\right],\left[f_{1}\right]\right) \mapsto \eta\left(f_{0}, f_{1}\right)$ (defined in $\S 2$ ) and that $\eta_{u, l, b}(f):=\eta\left(f, f^{\prime}\right)$ for a fixed choice of $\left[f^{\prime}\right] \in \beta_{u, l}^{-1}(b)$. We suppress the choice of $\left[f^{\prime}\right]$ from the notation. For $\beta_{u, l}$ the situation is similar and is discussed in the remark immediately following [CS16, Theorem 1.3].

Parts (a) and (b) of Theorem 1.6 follow from [CS16, Theorem 1.3]. The new part of Theorem 1.6 is (c), which is proven in $\S 2$. Part (d) follows because by (c) im $\eta_{u, l, b}=2 \mathbb{Z}_{\widehat{d}} / \operatorname{im} \theta_{u, l, b}$.

We remark that Theorem 1.2 is not an immediate corollary of Theorem 1.6, cf. [CS16, Remarks 2.20.a and 2.24].

\footnotetext{
${ }^{1}$ Indeed, for each $x \in H_{3}$ and $y \in K_{u, l}$ we have $2 \bar{l} x \cap_{N} y=2 l(x, y) \equiv 2 l(y, x)=2 \bar{l} y \cap_{N} x \equiv \overline{\bar{d}} 0$. Hence $\operatorname{im}\left(2 \rho_{d} \bar{l}\right) \cap_{N} K_{u, l}=\{0\} \subset \mathbb{Z}_{d}$.
} 
Addendum 1.7. In the notation of Theorem 1.6, for each $a \in \mathbb{Z}_{12},(u, l) \in \operatorname{im}(\varkappa \times \lambda), b \in C_{u, l}$ and $f \in \beta_{u, l}^{-1}(b)$

$$
\eta_{u, l, b}(f \# a)=\eta_{u, l, b}(f)+[2 a] \in \frac{\mathbb{Z}_{\widehat{d}}}{\operatorname{im} \theta_{u, l, b}} .
$$

This follows from the definition of $\eta_{u, l, b}(\S 2)$ and [CS16, Lemma 4.3.b].

Corollary 1.8. For each $(u, l) \in \operatorname{im}(\varkappa \times \lambda)$ let $d:=\operatorname{div} u$. There is $f_{u, l} \in(\varkappa \times \lambda)^{-1}(u, l)$ such that for each $f \in(\varkappa \times \lambda)^{-1}(u, l)$ and $a, a^{\prime} \in \mathbb{Z}_{12}$, denoting $b:=\beta\left(f, f_{u, l}\right) \in C_{u, l}$ we have

(a) $f \# a=f \# a^{\prime} \Leftrightarrow a=a^{\prime}$, provided either

- $u=0$ and $\operatorname{div} b$ is divisible by 6 , or

- $u \neq 0,2 \rho_{d} \bar{l}=0$ and $u$ is divisible by $24 \operatorname{ord}(4 b)$;

(b) $f \# a=f \# a^{\prime} \Leftrightarrow a \equiv a^{\prime} \bmod 2 \operatorname{gcd}(\operatorname{div} b, 6), \quad$ provided $u=0$;

(c) $\quad f \# a=f \# a^{\prime} \Leftrightarrow a \equiv a^{\prime} \bmod \frac{\frac{u}{\operatorname{ord}(4 b)}}{\operatorname{gcd}(u, 2)},{ }^{2} \quad$ provided $u \neq 0$ and $2 \rho_{d} \bar{l}=0$.

Part (a) follows from Parts (b,c). Parts (b,c) are proven in $\S 3$. Cf. Remark 3.1 and $\S 7$ below.

Theorem 1.6 has the following restatement analogous to Theorem 1.2 and to [CS16, Corollary 2.13.b].

Corollary 1.9. Denote by $B_{0}\left(H_{3}\right)$ the group of symmetric bilinear forms $H_{3} \times H_{3} \rightarrow \mathbb{Z}$. Then there is a surjection

$$
\begin{gathered}
\tau: \mathbb{Z}_{12} \times H_{1} \times H_{2}^{D I F F} \times B_{0}\left(H_{3}\right) \rightarrow E^{7}(N) \quad \text { such that } \\
\tau(a, b, u, l)=\tau\left(a^{\prime}, b^{\prime}, u^{\prime}, l^{\prime}\right) \Leftrightarrow u=u^{\prime}, \quad l=l^{\prime}, \quad b-b^{\prime} \in K_{u, l_{u}} \quad \text { and } \quad a-a^{\prime} \in \operatorname{im} \eta_{u, l_{u}, b},
\end{gathered}
$$

where $l_{u}:=l+\lambda \tau(0,0, u, 0)$.

Acknowledgments. We would like to thank B. Owens for assistance with the literature on 4-manifolds. We would like to thank the Hausdorff Institute for Mathematics and the University of Bonn for their hospitality and support during the early stages of this project.

\section{Definition of the invariants}

In this paper we use conventions, notation and the following definitions of [CS16, $\S \S 2.1,4.1]$.

- $N$ is a closed connected orientable 4-manifold with torsion free $H_{1}$;

- $f, f_{0}, f_{1}: N \rightarrow S^{7}$ are embeddings;

- $C=C_{f}$ is the closure of the complement in $S^{7}$ to a sufficiently small tubular neighborhood of $f(N)$; the orientation on $C$ is inherited from the orientation of $S^{7}$;

$\partial \nu=\partial \nu_{f}: \partial C \rightarrow N$ is the sphere subbundle of the normal vector bundle of $f$ : the total space of $\partial \nu$ is identified with $\partial C$.

Consider the following diagram:

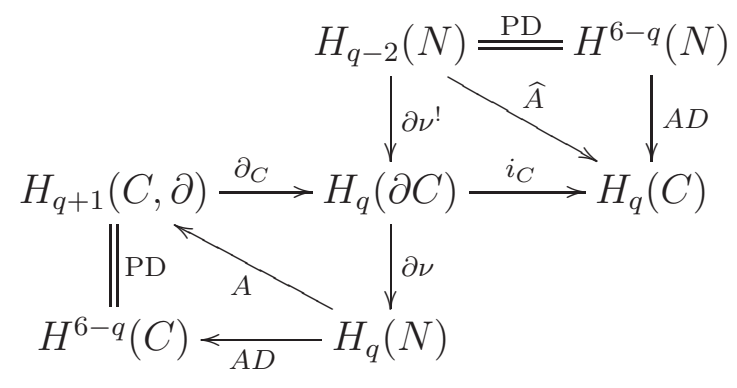

\footnotetext{
${ }^{2}$ The class $u$ is divisible by $d$ and hence by the order ord(4b) of $d$ in the $d$-group $C_{u, l}$.
} 
Here $A D$ is Alexander duality and $A=A_{f}, \widehat{A}=\widehat{A}_{f}$ are homology Alexander duality isomorphisms.

Define

$$
\varkappa[f]:=A_{f}^{-1}\left(A_{f}[N] \cap_{C_{f}} A_{f}[N]\right) \in H_{2}
$$

[CS16, Lemma 3.2. $\left.\varkappa^{\prime}\right]$. Define

$$
\lambda[f](x, y):=x \cap_{N} \widehat{A}_{f}^{-1}\left(A_{f}[N] \cap_{C_{f}} \widehat{A}_{f} y\right)
$$

for each $x, y \in H_{3}$ [CS16, Lemma 3.2. $\left.\lambda^{\prime}\right]$.

We abbreviate the subscript $f_{k}$ to just $k$. For a bundle isomorphism $\varphi: \partial C_{0} \rightarrow \partial C_{1}$ define a closed oriented 7-manifold $M=M_{\varphi}:=C_{0} \cup_{\varphi}\left(-C_{1}\right)$. We call a bundle isomorphism $\varphi$ : $\partial C_{0} \rightarrow \partial C_{1}$ a $\pi$-isomorphism if $M_{\varphi}$ is parallelizable. We shall omit the phrase 'for a bundle isomorphism $\varphi^{\prime}$ if its choice is clear from the context.

If $P$ is a (compact oriented) codimension $c$ submanifold of a manifold $Q$ and either $y \in H_{k}(Q)$ or $y \in H_{k}(Q, \partial)$, denote

$$
r_{P, Q}(y)=r_{P}(y)=y \cap P:=\operatorname{PD}\left(\left.(\operatorname{PD} y)\right|_{P}\right) \in H_{k-c}(P, \partial) .
$$

A class $Y \in H_{5}\left(M_{\varphi}\right)$ is a joint Seifert class if $Y \cap C_{k}=A_{k}[N]$ for each $k=0,1$ [CS16, Lemma 3.13.a]. A joint Seifert class $Y \in H_{5}\left(M_{\varphi}\right)$ is called a $d$-class for an integer $d$ if $\rho_{d} Y^{2}=0$ (or, equivalently, $\left.Y^{2} \in d H_{3}\left(M_{\varphi}\right)\right)$.

Assume that $\varkappa\left(f_{0}\right)=\varkappa\left(f_{1}\right)$ and that $\lambda\left(f_{0}\right)=\lambda\left(f_{1}\right)$. Denote $d:=\operatorname{div} \varkappa\left(f_{0}\right)$. By [CS16, Lemmas 2.4 and 2.5] there is a $\pi$-isomorphism $\varphi: \partial C_{0} \rightarrow \partial C_{1}$ and a joint Seifert class $Y \in$ $H_{5}\left(M_{\varphi}\right)$. Define

$$
\beta\left(f_{0}, f_{1}\right):=\left[\left(i_{\partial C_{0}, M_{\varphi}} \partial \nu_{0}^{!}\right)^{-1} \rho_{d} Y^{2}\right] \in C_{\varkappa\left(f_{0}\right), \lambda\left(f_{0}\right)}
$$

using the composition $H_{1}\left(N ; \mathbb{Z}_{d}\right) \stackrel{\partial \nu_{0}^{!}}{\longrightarrow} H_{3}\left(\partial C_{0} ; \mathbb{Z}_{d}\right) \stackrel{i_{\partial C_{0}, M_{\varphi}}}{\longrightarrow} H_{3}\left(M_{\varphi} ; \mathbb{Z}_{d}\right)$.

Definition of $\eta(\varphi, Y)$ for a $\pi$-isomorphism $\varphi: \partial C_{0} \rightarrow \partial C_{1}$ and a $d$-class $Y \in H_{5}\left(M_{\varphi}\right)$, of $Y_{f, y}$ and $\theta(f, y)$. Since $\varphi: \partial C_{0} \rightarrow \partial C_{1}$ is a $\pi$-isomorphism, $M_{\varphi}$ is spin. Take any normal spin structure on $M$ given by [CS16, Lemma 4.2]. Since $M_{\varphi}$ is simply-connected, a normal spin structure on $M_{\varphi}$ is unique. Since $\Omega_{7}^{\text {Spin }}\left(\mathbb{C} P^{\infty}\right)=0[\mathrm{KS} 91$, Lemma 6.1] there is a 8-manifold $W$ with a normal spin structure and $z \in H_{6}(W, \partial)$ such that $\partial W \underset{\text { spin }}{=} M_{\varphi}$ and $\partial z=Y$. Consider the following fragment of the exact sequence of the pair $(W, \partial W)$ :

$$
H_{4}\left(\partial W ; \mathbb{Z}_{d}\right) \stackrel{i_{W}}{\rightarrow} H_{4}\left(W ; \mathbb{Z}_{d}\right) \stackrel{j_{W}}{\rightarrow} H_{4}\left(W, \partial ; \mathbb{Z}_{d}\right) \stackrel{\partial_{W}}{\rightarrow} H_{3}\left(\partial W ; \mathbb{Z}_{d}\right) .
$$

Since $\partial_{W} \rho_{d} z^{2}=\rho_{d} Y^{2}=0$, there is a class $\overline{z^{2}} \in H_{4}\left(W ; \mathbb{Z}_{d}\right)$ such that $j_{W} \overline{z^{2}}=\rho_{d} z^{2}$. Denote by $p_{W}^{*} \in H_{4}(W, \partial)$ the spin characteristic class [CS16, §3.1]. Define

$$
\eta(\varphi, Y)=\eta\left(f_{0}, f_{1}, d, \varphi, Y\right):=\rho_{\widehat{d}}\left(\overline{z^{2}} \cap_{W}\left(z^{2}-p_{W}^{*}\right)\right) \in \mathbb{Z}_{\widehat{d}}
$$

For $y \in H_{3}$ denote

$$
Y_{f, y}:=\partial\left(A_{f}[N] \times I\right)+i \widehat{A}_{f} y \in H_{5}\left(C_{f} \times I\right) \quad \text { and } \quad \theta(f, y):=\eta\left(\operatorname{id} \partial C_{f}, Y_{f, y}\right) \in \mathbb{Z}_{\widehat{d}} .
$$

Lemma 2.1 (proved in $\S 4, \S 5)$. (a) For every $f$ and $y, \theta(f, y)$ is divisible by 4.

(b) The map $\theta(f, \cdot): K_{\varkappa(f), \lambda(f)} \lambda(f) \rightarrow \mathbb{Z}_{\widehat{d}}$ is a homomorphism, where $d:=\operatorname{div} \varkappa(f)$.

(c) For every $\left[f_{0}\right],\left[f_{1}\right] \in(\varkappa \times \lambda)^{-1}(u, l)$ and $y \in K_{u, l}$, we have for $d:=\operatorname{div} u$ that $\theta\left(f_{0}, y\right)-$ $\theta\left(f_{1}, y\right)=4 \rho_{\widehat{d}}\left(\beta\left(f_{0}, f_{1}\right) \cap_{N} y\right)$. 
Definition of $\theta_{u, l, b}$. Take any $(u, l) \in \operatorname{im}(\varkappa \times \lambda)$ and $b \in C_{u, l}$. Let $d:=\operatorname{div} u$. Define

$$
\theta_{u, l, b}: K_{u, l} \rightarrow 4 \mathbb{Z}_{\widehat{d}} \quad \text { by } \quad \theta_{u, l, b}(y):=\theta(f, y), \quad \text { where } \quad[f] \in \beta_{u, l}^{-1}(b) .
$$

The map $\theta_{u, l, b}$ is well-defined (i.e. is independent of the choice of $f$ ) and is a homomorphism by Lemma 2.1.ab and the transitivity of $\beta$ [CS16, Lemma 2.10].

Definition of $\eta\left(f_{0}, f_{1}\right)$. Take representatives $f_{0}, f_{1}$ of two isotopy classes in $(\varkappa \times \lambda)^{-1}(u, l)$ such that $\beta\left(f_{0}, f_{1}\right)=0$. By [CS16, Lemma 2.5] there is a $\pi$-isomorphism $\varphi: \partial C_{0} \rightarrow \partial C_{1}$. By [CS16, Lemma 4.1] there is a $d$-class $Y \in H_{5}\left(M_{\varphi}\right)$ for $d:=\operatorname{div} \varkappa\left(f_{0}\right)$. Define

$$
\eta\left(f_{0}, f_{1}\right):=[\eta(\varphi, Y)] \in \frac{\mathbb{Z}_{\widehat{d}}}{\operatorname{im} \theta_{u, l, b}} .
$$

This is well-defined by [CS16, Lemma 4.3.c] and Lemma 2.3.a below, and is even by [CS16, Lemma 4.3.a].

Lemma 2.2. Let $f_{0}, f_{1}, f_{2}: N \rightarrow S^{7}$ be embeddings and $\varphi_{01}: \partial C_{0} \rightarrow \partial C_{1}, \varphi_{12}: \partial C_{1} \rightarrow \partial C_{2}$ $\pi$-isomorphisms and $Y_{01} \in H_{5}\left(M_{\varphi_{01}}\right), Y_{12} \in H_{5}\left(M_{\varphi_{12}}\right)$ d-classes. Then $\varphi_{02}:=\varphi_{12} \varphi_{01}$ is a $\pi$-isomorphism and there is a d-class $Y_{02} \in H_{5}\left(M_{\varphi_{02}}\right)$ such that $\eta\left(\varphi_{02}, Y_{02}\right)=\eta\left(\varphi_{01}, Y_{01}\right)+$ $\eta\left(\varphi_{02}, Y_{12}\right)$.

This is proved analogously to [CS11, Lemma 2.10], cf. [Sk08', §2, Additivity Lemma] (the property that $Y_{02}$ is a $d$-class is achieved analogously to [CS16, 44.3 , proof of Lemma 4.6]).

Lemma 2.3. Let $\left[f_{0}\right],\left[f_{1}\right] \in(\varkappa \times \lambda)^{-1}(u, l)$ be such that $\beta\left(f_{0}, f_{1}\right)=0$. Denote $d:=\operatorname{div} u$. Take any $\pi$-isomorphism $\varphi: \partial C_{0} \rightarrow \partial C_{1}$.

(a) The residue $\eta\left(f_{0}, f_{1}\right)$ is independent of the choice of a d-class $Y \in H_{5}\left(M_{\varphi}\right)$.

(b) If $\eta\left(f_{0}, f_{1}\right)=0$, then there is a d-class $Y \in H_{5}\left(M_{\varphi}\right)$ such that $\eta(\varphi, Y)=0 \in \mathbb{Z}_{\widehat{d}}$.

Proof of (a). Take any pair of $d$-classes $Y^{\prime}, Y^{\prime \prime} \in H_{5}\left(M_{\varphi}\right)$. Part (a) follows because

$$
\eta\left(\varphi, Y^{\prime}\right)-\eta\left(\varphi, Y^{\prime \prime}\right) \stackrel{(1)}{=} \eta\left(\operatorname{id} \partial C_{0}, Y\right) \stackrel{(2)}{=} \theta\left(f_{0}, y\right)=\theta_{u, l, \beta\left(f_{0}, f^{\prime}\right)}(y) \in \mathbb{Z}_{\widehat{d}}
$$

where

- equality (1) holds for some $d$-class $Y \in H_{5}\left(M_{f_{0}}\right)$ by Lemma 2.2 ;

- equality (2) holds for some $y \in K_{u, l}$ by the description of $d$-classes [CS16, Lemma 4.7].

Proof of (b). Part (b) follows because

$$
0 \stackrel{(1)}{=} \eta\left(\varphi, Y^{\prime}\right)-\theta_{u, l, \beta_{u, l}\left(f_{0}\right)}(y)=\eta\left(\varphi, Y^{\prime}\right)-\theta\left(f_{0}, y\right) \stackrel{(3)}{=} \eta(\varphi, Y) \in \mathbb{Z}_{\widehat{d}}
$$

where

- equality (1) holds for some $d$-class $Y^{\prime} \in H_{5}\left(M_{\varphi}\right)$ and $y \in K_{u, l}$ because $\eta\left(f_{0}, f_{1}\right)=0$;

- equality (3) holds for some $d$-class $Y \in H_{5}\left(M_{\varphi}\right)$ by Lemma 2.2.

Lemma 2.4 (Transitivity of $\eta$ ). For any triple of embeddings $f_{0}, f_{1}, f_{2}: N \rightarrow S^{7}$ having the same values of $\varkappa$-and $\lambda$-invariants and the property that that $\beta\left(f_{0}, f_{1}\right)=\beta\left(f_{1}, f_{2}\right)=0$, we have $\eta\left(f_{2}, f_{0}\right)=\eta\left(f_{2}, f_{1}\right)+\eta\left(f_{1}, f_{0}\right)$.

This follows by Lemma 2.2.

Theorem 2.5 (Isotopy classification). If $\lambda\left(f_{0}\right)=\lambda\left(f_{1}\right), \varkappa\left(f_{0}\right)=\varkappa\left(f_{1}\right), \beta\left(f_{0}, f_{1}\right)=0$ and $\eta\left(f_{0}, f_{1}\right)=0$, then $f_{0}$ is isotopic to $f_{1}$. 
Proof. The proof is analogous to the proof of [CS16, Isotopy Classification Modulo Knots Theorem 2.8]. We only need to replace the second paragraph of that proof by the following sentence: 'Since $\eta\left(f_{0}, f_{1}\right)=0$, by Lemma 2.3.b we can change $Y$ and assume additionally that $\eta(\varphi, Y)=0$ '’

Definition of $\eta_{u, l, b}$. Take any $\left[f_{0}\right] \in \beta_{u, l}^{-1}(b)$. Define the map

$$
\eta_{u, l, b}: \beta_{u, l}^{-1}(b) \rightarrow \frac{\mathbb{Z}_{\widehat{d}}}{\operatorname{im} \theta_{u, l, b}} \quad \text { by } \quad \eta_{u, l, b}[f]:=\eta\left(f, f_{0}\right) .
$$

The map $\eta_{u, l, b}$ depends on $f_{0}$ but we do not indicate this in the notation.

Proof of Theorem 1.6.c. The property on $\theta_{u, l, b}-\theta_{u, l, b^{\prime}}$ holds by Lemma 2.1.c. The map $\eta_{u, l, b}$ is injective by the Isotopy Classification Theorem 2.5. The image of this map consists of all even elements by [CS16, Lemma 4.3.a] and Addendum 1.7.

\section{Proof of Corollaries 1.4 and 1.8.bc}

Proof of Corollary 1.4. By Theorem 1.6.c and Addendum $1.7(i i) \Rightarrow(i)$.

The other direction is implied by the following assertions.

(*) If the action of knots is free and $H_{1}$ is torsion free, then $H_{1}=0$.

(**) If the action of knots is free and $H_{1}=0$, then $H_{2}=0$.

Proof of $\left(^{*}\right)$. By Theorem 1.6.a there is an embedding $f_{1}: N \rightarrow S^{7}$ and $\left(u, l_{1}\right):=(\varkappa \times \lambda)\left(f_{1}\right)$ is a symmetric pair. If $H_{1} \neq 0$, then there is a basis $\left\{y_{1}, \ldots, y_{n}\right\}$ for $H_{3}$ with $n>0$. Express $l_{1}=l_{1}^{i j}$ as a matrix with respect to this basis. For any symmetric matrix $a^{i j}$ the pair $\left(u, l_{1}-a\right)$ is again a symmetric pair. Take $a^{i j}$ to be the symmetric matrix with $a^{i j}=l_{1}^{i j}$ for $i \leq j$. Then $l:=l_{1}-a$ is strictly upper-triangular with respect to the chosen basis; i.e. $l^{i j}=0$ for $i \geq j$. The pair $(u, l)$ is symmetric and $l\left(y_{1}\right)=0$. By Theorem 1.6.a there is an embedding $f$ with $(\varkappa \times \lambda)(f)=(u, l)$.

Since $y_{1} \in K_{u, l}$ is primitive, by Poincaré duality there is $x \in H_{1}$ such that $x \cap_{N} y_{1}=1$. Since the action of knots is free, by Theorem 1.6.c and Addendum $1.7 u$ is divisible by 24 and $\theta_{u, l, b}=0$ for every $b \in C_{u, l}$. Then $d:=\operatorname{div} u$ is divisible by 24 , and so is $\widehat{d}$. Hence by Theorem 1.6.c

$$
0-0=\theta_{u, l, b+[x]}\left(y_{1}\right)-\theta_{u, l, b}\left(y_{1}\right)=4 \rho_{\widehat{d}}\left(x \cap_{N} y_{1}\right)=4 \neq 0 \in \mathbb{Z}_{\widehat{d}} .
$$

This contradiction shows that $H_{1}=0$.

Proof of $\left(^{* *}\right)$. Since the action of knots is free, by Theorem 1.6.c and Addendum 1.7 every element $u \in H_{2}^{D I F F}$ is divisible by 24. Since $\rho_{2} u=\operatorname{PD} w_{2}(N)$, we obtain $w_{2}(N)=0$, so the intersection form $\cap_{N}$ of $N$ is even. If $H_{2} \neq 0$, then the intersection form of $N$ is indefinite [Do87, Theorem 1]. Hence by [GS99, Theorem 1.2.21] this form is isomorphic to $m H_{+} \oplus n E_{8}$, where $H_{+}$is the standard hyperbolic form with matrix $\left(\begin{array}{ll}0 & 1 \\ 1 & 0\end{array}\right)$, and the form $E_{8}$ is positive definite, so $m>0$. By [GS99, Lemma 1.2.20] $\sigma(N)$ is divisible by 4 . Then $u:=(2, \sigma(N) / 2) \oplus(m-1) 0 \oplus n 0 \in H_{2}^{D I F F}$ and $u$ is not divisible by 24 . This contradiction shows that $H_{2}=0$.

Remark 3.1. In Corollary 1.8.bc each of the assumptions ' $u=0$ ' and ' $u \neq 0$ and $2 \rho_{d} \bar{l}=0$ ' can be replaced by each of the following successively weaker assumptions:

(1) $\rho_{\widehat{d}} K_{u, l} \subset \rho_{\widehat{d}} H_{3}$ is a direct summand, or

(2) every homomorphism $\rho_{\widehat{d}} K_{u, l} \rightarrow 4 \mathbb{Z}_{\widehat{d}}$ extends to $\rho_{\widehat{d}} H_{3}$, or

(3) there is an element $\widetilde{b} \in C_{u, l}$ such that $\theta_{u, l, \widetilde{b}}=0$. 
Clearly, 'either $u=0$ or $2 \rho_{d} \bar{l}=0^{\prime} \Rightarrow(1) \Rightarrow(2)$.

Proof that $(2) \Rightarrow(3)$. Take any $b^{\prime} \in C_{u, l}$. We have $\theta_{u, l, b^{\prime}}=\theta_{u, l, b^{\prime}}^{+} \rho_{\widehat{d}}$ for some homomorphism $\theta_{u, l, b^{\prime}}^{+}: \rho_{\widehat{d}} K_{u, l} \rightarrow 4 \mathbb{Z}_{\widehat{d}}$. Extend $\theta_{u, l, b^{\prime}}^{+}$to a homomorphism $\rho_{\widehat{d}} H_{3} \rightarrow 4 \mathbb{Z}_{\widehat{d}}$. Since $H_{3}$ is free, $\rho_{\widehat{d}} H_{3}$ is a free $\mathbb{Z}_{\widehat{d}}$ module. Hence the latter homomorphism is divisible by 4 . Then by Poincaré duality there is a class $x \in \rho_{\widehat{d}} H_{1}$ such that $\theta_{u, l, b^{\prime}}^{+}(z)=4 x \cap_{N} z$ for every $z \in \rho_{\widehat{d}} K_{u, l}$. Let $\widetilde{b}:=b^{\prime}+[\widetilde{x}]$, where $\widetilde{x} \in \rho_{d} H_{1}$ is a lifting of $x$. Then by Theorem 1.6

$$
\theta_{u, l, \widehat{b}}(y)=\theta_{u, l, b^{\prime}}(y)-4 \rho_{\widehat{d}}\left([\widetilde{x}] \cap_{N} y\right)=\theta_{u, l, b^{\prime}}^{+}\left(\rho_{\widehat{d}} y\right)-4 x \cap_{N} \rho_{\widehat{d}} y=0 \quad \text { for every } \quad y \in K_{u, l} .
$$

Proof of Corollary 1.8.bc under the assumption (3) of Remark 3.1. Define the element $\beta_{u, l}^{\prime}(f):=$ $\widetilde{b}-\beta_{u, l}(f) \in C_{u, l}$. Then $\theta_{u, l, b}^{\prime}=\theta_{u, l, \tilde{b}-b}$ for every $b \in C_{u, l}$, hence $\theta_{u, l, 0}^{\prime}=0$. Therefore we may assume that $\beta_{u, l}$ is chosen so that $\theta_{u, l, 0}=0$.

Take any $b \in C_{u, l}$ and denote $K:=4 b \cap_{d} K_{u, l} \subset \mathbb{Z}_{d}$. So

$$
\operatorname{gcd}(d, 2) \cdot\left|\beta_{u, l}^{-1}(b)\right| \stackrel{(1)}{=} \frac{\widehat{d}}{\left|\operatorname{im} \theta_{u, l, b}\right|} \stackrel{(2)}{=} \frac{\widehat{d}}{\left|\rho_{\widehat{d}} K\right|}=\left[\mathbb{Z}_{\widehat{d}}: \rho_{\widehat{d}} K\right]=\operatorname{gcd}\left(\widehat{d},\left[\mathbb{Z}_{d}: K\right]\right)=\left[\widehat{\mathbb{Z}_{d}: K}\right],
$$

where equalities (1) and (2) hold by Theorem 1.6.d. Now Corollary 1.8.bc is implied by Addendum 1.7 and the following Lemma 3.2.

Lemma 3.2. Let $V$ be a free $\mathbb{Z}$-module, $d$ an integer, $\rho_{d}: V \rightarrow V / d V$ the reduction $\bmod d$ and $m: V \rightarrow V^{*}$ a homomorphism whose polarization $V \times V \rightarrow \mathbb{Z}$ has a symmetric mod d reduction. Then

(a) a bilinear map $\cap_{d}: \operatorname{coker}\left(\rho_{d} m\right) \times \operatorname{ker}\left(\rho_{d} m\right) \rightarrow \mathbb{Z}_{d}$ is well-defined by $[x] \cap_{d} y:=\rho_{d} x(y)$ for $x \in V^{*}$.

(b) for every $c \in \operatorname{coker}\left(\rho_{d} m\right)$

$$
\left[\mathbb{Z}_{d}: c \cap_{d} \operatorname{ker}\left(\rho_{d} m\right)\right]= \begin{cases}\operatorname{div} c & d=0 \\ \frac{d}{\operatorname{ord} c} & d \neq 0\end{cases}
$$

This lemma is elementary and so possibly known. Part (a) is simple and is essentially proved in footnote 1.

Proof of part (b) for $d=0$. We need to prove the following:

Let $V$ be a free $\mathbb{Z}$-module and $m: V \rightarrow V^{*}$ a homomorphism whose polarization $V \times V \rightarrow \mathbb{Z}$ is symmetric. Then for every $c \in \operatorname{coker} m$ we have $[\mathbb{Z}: c(\operatorname{ker} m)]=\operatorname{div} c$.

Assume that $q$ is a divisor of $c+$ Tors coker $m$. Then there exist $s \in \mathbb{Z}, l_{1}, \ldots, l_{s} \in \mathbb{Z}-\{0\}$ and $c_{0}, t_{1}, \ldots, t_{s} \in V^{*}$ such that $c=q c_{0}+t_{1}+\ldots+t_{s}$ and $l_{n} t_{n} \in \operatorname{im} m$ for every $n=1, \ldots, s$. Take any $y \in \operatorname{ker} m$. Since the polarization of $m$ is symmetric, we have $l_{n} t_{n}(y)=0 \in \mathbb{Z}$, thus $t_{n}(y)=0$. Hence $c(y)=q c_{0}(y)+\left(t_{1}+\ldots+t_{s}\right)(y)=q c_{0}(y)$ is divisible by $q$. Thus $[\mathbb{Z}: c($ ker $m)]$ is divisible by $q$.

Assume that $q$ is a divisor of $[\mathbb{Z}: c(\operatorname{ker} m)]$. Then $\left.c\right|_{\operatorname{ker} m}$ is divisible by $q$. The subgroup ker $m \subset V$ is a direct summand. Take a decomposition $V=\operatorname{ker} m \oplus V^{\prime}$. Since $\left.m\right|_{V^{\prime}}: V^{\prime} \rightarrow \operatorname{im} m$ is an isomorphism, there is an element $x \in V^{\prime}$ such that $\left.c\right|_{V^{\prime}}=\left.m(x)\right|_{V^{\prime}}$. Since the polarization of $m$ is symmetric, $\left.m(x)\right|_{\operatorname{ker} m}=0$. Then $c-m(x)$ coincides with $c$ on ker $m$ and is zero on $V^{\prime}$. So $c-m(x)=q c_{0}$ for some $c_{0} \in V^{*}$. Thus $c+$ Tors coker $m$ is divisible by $q$. 
Proof of part (b) for $d \neq 0$. We need to prove that $\left|c\left(\operatorname{ker}\left(\rho_{d} m\right)\right)\right|=\operatorname{ord} c$ for every $c \in \operatorname{coker}\left(\rho_{d} m\right)$. (We remark that this is obvious for $\rho_{d} m=0$ and this case is sufficient for Corollary 1.8.c.)

Denote $K:=\rho_{d} \operatorname{ker}\left(\rho_{d} m\right) \subset V / d V$. Since the polarization $V \times V \rightarrow \mathbb{Z}$ of $m$ has a symmetric mod $d$ reduction, $\operatorname{im}\left(\rho_{d} m\right) \subset K^{\perp} \subset V^{*} / d V^{*}$. Since $\left|\operatorname{im}\left(\rho_{d} m\right)\right|=\frac{|V / d V|}{|K|}=\left|K^{\perp}\right|$, it follows that $\operatorname{im}\left(\rho_{d} m\right)=K^{\perp}$. Now the required assertion follows because for every $c^{\prime} \in V^{*} / d V^{*}$

$$
\left|c^{\prime}(K)\right|=\frac{d}{\operatorname{div} c^{\prime}(K)}=\min \left\{r \mid r c^{\prime}(K)=0\right\}=\min \left\{r \mid r c^{\prime} \in K^{\perp}\right\}=\operatorname{ord}_{\left(V^{*} / d V^{*}\right) / K^{\perp}}\left(c^{\prime}+K^{\perp}\right) .
$$

\section{Proof of Lemma 2.1.b}

Before reading the proof of Lemma 2.1 we recommend reading the idea of the proof in $\S 6$.

In this and the following section $l=\lambda(f)=\lambda\left(f_{0}\right)=\lambda\left(f_{1}\right), u=\varkappa(f)=\varkappa\left(f_{0}\right)=\varkappa\left(f_{1}\right)$ and $d=\operatorname{div} u$. Denote $1_{m}:=(1,0, \ldots, 0) \in S^{m}, \Delta:=1_{2} \times D^{4} \times 1_{1}$ and $t:=S^{2} \times 0 \times S^{2}$. For every $y \in H_{3}$ take the following objects constructed in [CS16, Proof of Lemma 4.8]: 6-manifolds $V \subset C_{f}$ and $\widehat{V}:=V \cup_{S^{2} \times S^{3}}\left(S^{2} \times D^{4} \times 1\right)$, an embedding $v_{2}: S^{2} \times S^{3} \times D^{2} \rightarrow \operatorname{Int} C_{f}=\operatorname{Int} C_{f} \times \frac{1}{2}$, 8-manifolds $W_{-} \subset C_{f} \times I$ and $W:=W_{-} \cup_{S^{2} \times S^{3} \times S^{2}}\left(S^{2} \times D^{4} \times S^{2}\right)$, classes $Z \in H_{6}(W, \partial)$ and $z:=Z+[\widehat{V}] \in H_{6}(W, \partial)$. The objects are not uniquely constructed from $y$, and we allow arbitrary choices in that construction.

Definition of $W^{\prime}, W_{-}^{\prime}$ and $i^{\prime}: W^{\prime} \rightarrow W$. Let

$$
W_{-}^{\prime}:=C_{f}-\operatorname{Intim} v_{2} \text { and } W^{\prime}:=W_{-}^{\prime} \cup_{\left.v_{2}\right|_{S^{2} \times S^{3} \times S^{1}}} S^{2} \times D^{4} \times S^{1}
$$

(the manifold $W^{\prime}$ may be called the result of an $S^{1}$-parametric surgery along $v_{2}$.) Define an embedding $W_{-}^{\prime} \rightarrow W_{-}$by $x \mapsto x \times 1 / 2$. We assume that this embedding and the standard embedding $S^{2} \times D^{4} \times S^{1} \rightarrow S^{2} \times D^{4} \times S^{2}$ (that is the product of the identity and the equatorial inclusion $S^{1} \rightarrow S^{2}$ ) fit together to give an embedding

$$
i^{\prime}: W^{\prime} \rightarrow W
$$

Observe that $\Delta, \widehat{V} \subset W^{\prime}$.

Lemma 4.1. For every $y \in H_{3}$ we have

$$
z^{2} \cap_{W} W_{-} \equiv 2 i_{V, W_{-}}(Z \cap V) \in H_{4}\left(W_{-}, \partial\right)
$$

(since $\partial V \subset \partial W_{-}$, the inclusion induces a map $i_{V, W_{-}}: H_{4}(V, \partial) \rightarrow H_{4}\left(W_{-}, \partial\right)$ ).

Proof. Since $\widehat{V} \subset W^{\prime}$, we have $[\widehat{V}]^{2}=0 \in H_{4}(W, \partial)$. Also

$$
Z^{2} \cap W_{-}=\left(A_{f}[N] \times I\right)^{2} \cap W_{-}=A_{f} \varkappa(f) \times I \cap W_{-} \equiv 0 \in H_{4}\left(W_{-}, \partial\right) .
$$

Hence

$$
z^{2} \cap W_{-}=(Z+[\widehat{V}])^{2} \cap W_{-} \equiv 2\left(Z \cap_{W}[\widehat{V}]\right) \cap W_{-}=2 i_{V, W_{-}}\left(Z \cap \widehat{V} \cap W_{-}\right)=2 i_{V, W_{-}}(Z \cap V) .
$$


Proof of Lemma 2.1.b. In this proof a statement or a construction involving $k$ holds or is made for $k=0,1$. Given $y_{k} \in K_{u, l}$ construct the manifold $W_{k}$ as $W$ of [CS16, Proof of Lemma 4.8] by parametric surgery in $C_{f} \times[k-1, k]$. We add the subscript $k$ to $W_{-}, W_{-}^{\prime}, t, \Delta, Z, \widehat{V}, z$ constructed in [CS16, Proof of Lemma 4.8]. (So unlike in other parts of this paper, a subscript 0 for a manifold does not mean deletion of a codimension 0 ball from the manifold.) Define

$$
W:=W_{0} \cup_{C_{f} \times 0} W_{1} \quad \text { and } \quad W_{-}:=C_{f} \times[-1,1]-\operatorname{Int} \operatorname{im}\left(v_{3,0} \sqcup v_{3,1}\right)=W_{0-} \cup_{C_{f} \times 0} W_{1-} .
$$

The manifold $W$ just defined should not be confused with the manifolds which were previously denoted $W$ but are now denoted $W_{0}$ and $W_{1}$. The same remark holds for $W_{-}$and for $Z, V, \widehat{V}, z$ constructed below.

The spin structure on $W_{-}$coming from $S^{7} \times[-1,1]$ extends to $W$. Clearly, we have $\partial W \underset{\text { spin }}{=} \partial\left(C_{f} \times[-1,1]\right) \underset{\text { spin }}{\cong} M_{f}$ (for the 'boundary' spin structures on $\partial\left(C_{f} \times[-1,1]\right)$ and on $\left.M_{f}\right)$.

Since $H_{5}\left(t_{k} \times \Delta_{k}\right)=0$, by the cohomological exact sequence of the pair $\left(W, W_{-}\right)$(cf. diagram $(*)$ in [CS16, Proof of Lemma 4.8]), $r_{W_{-}}: H_{6}(W, \partial) \rightarrow H_{6}\left(W_{-}, \partial\right)$ is an epimorphism. Take any

$$
Z \in r_{W_{-}}^{-1}\left(A_{f}[N] \times[-1,1] \cap W_{-}\right) \subset H_{6}(W, \partial)
$$

Denote

$$
V:=V_{0} \sqcup V_{1}, \quad \widehat{V}:=\widehat{V}_{0} \sqcup \widehat{V}_{1} \quad \text { and } \quad z:=Z+[\widehat{V}] \in H_{6}(W, \partial) .
$$

Since $\partial_{W} Z=Y_{f, 0}$ and $\partial_{W}\left[\widehat{V}_{k}\right]=i_{\partial W} \widehat{A}_{f} y_{k}$, we have $\partial z=Y_{f, y_{0}+y_{1}}$. Thus the pair $(W, z)$ is a spin null-bordism of $\left(M_{f}, Y_{f, y_{0}+y_{1}}\right)$.

Since $y_{k} \in K_{u, l}$, we have $\partial z_{k}^{2} \equiv \overline{\bar{d}} 0$. Take any $\overline{z_{k}^{2}} \in j_{W_{k}}^{-1} \rho_{d} z_{k}^{2}$. Let

$$
\overline{z^{2}}:=i_{W_{0}, W} \overline{z_{0}^{2}}+i_{W_{1}, W} \overline{z_{1}^{2}}
$$

Then $\overline{z^{2}} \cap W_{k}=\overline{z_{k}^{2}}$. Also

$$
\begin{gathered}
j_{W} \overline{z^{2}} \cap W_{-}=\sum_{k=0}^{1} j_{W_{k}} \overline{z_{k}^{2}} \cap W_{-}=\rho_{d} \sum_{k=0}^{1} z_{k}^{2} \cap W_{k-} \quad \text { and } \\
\sum_{k=0}^{1} z_{k}^{2} \cap W_{k-} \stackrel{(1)}{=} 2 \sum_{k=0}^{1} i_{V_{k}, W_{k-}}\left(Z_{k} \cap V_{k}\right)=2 i_{V, W_{-}}(Z \cap V) \stackrel{(3)}{=} z^{2} \cap W_{-} .
\end{gathered}
$$

Here the congruences (1) and (3) modulo $d$ hold by Lemma 4.1 and analogously to Lemma 4.1, respectively.

Hence by the cohomological exact sequence of the pair $\left(W, W_{-}\right)$with coefficients $\mathbb{Z}_{d}$ (cf. dia$\operatorname{gram}\left(^{*}\right)$ in $\left[\mathrm{CS} 16\right.$, Proof of Lemma 4.8]) $j_{W} \overline{z^{2}}-\rho_{d} z^{2}=n_{0}\left[t_{0}\right]+n_{1}\left[t_{1}\right]$ for some $n_{0}, n_{1} \in \mathbb{Z}_{d}$. We have

$$
n_{k}\left[t_{k}\right]=\left(j_{W} \overline{z^{2}}-\rho_{d} z^{2}\right) \cap W_{k}=j_{W_{k}} \overline{z_{k}^{2}}-\rho_{d} z_{k}^{2}=0 \in H_{4}\left(W_{k}, \partial ; \mathbb{Z}_{d}\right) .
$$

Therefore $n_{0}=n_{1}=0$. So $j_{W} \overline{z^{2}}=\rho_{d} z^{2}$.

Since $\widetilde{W_{k}}:=W_{k}-C_{f} \times[0,(2 k-1) / 3)$ is a deformation retract of $W_{k}$, the inclusion $\widetilde{W_{k}} \rightarrow W_{k}$ induces an isomorphism on $H_{4}$. Clearly, $z \cap W_{k}=z_{k}$, so $z^{2} \cap W_{k}=z_{k}^{2}$. Hence

$$
\overline{z^{2}} \cap_{W}\left(z^{2}-p_{W}^{*}\right)=\sum_{k=0}^{1}\left(\overline{z^{2}} \cap W_{k}\right) \cap_{W_{k}}\left(\left(z^{2}-p_{W}^{*}\right) \cap W_{k}\right)=\sum_{k=0}^{1} \overline{z_{k}^{2}} \cap_{W_{k}}\left(z_{k}^{2}-p_{W_{k}}^{*}\right) .
$$

So $\eta(f, \cdot)$ is a homomorphism. 


\section{Proof of Lemma 2.1.ac}

Lemma 5.1. For every $y \in H_{3}$ we have:

(a) $\partial(Z \cap V)=[\partial \Delta]-i_{\partial C_{f}, \partial V} \xi y \in H_{3}(\partial V)$, where $\xi: N_{0} \rightarrow \partial C_{f}$ is a weakly unlinked section for $f$ (see definition in [CS16, §2.2]);

(b) $p_{W}^{*}=2 m[t] \in H_{4}(W, \partial)$ for some $m \in \mathbb{Z}$.

Lemma 5.1.b is essentially proved in the proof of [CS16, Lemma 4.8].

Proof of (a). The equality follows because

$$
\begin{gathered}
Z \cap V=\left(Z \cap W_{-}\right) \cap V=\left(A_{f}[N] \times I\right) \cap V=A_{f}[N] \cap V \in H_{4}(V, \partial) \quad \text { and } \\
\partial\left(A_{f}[N] \cap V\right)=A_{f}[N] \cap \partial V=i_{\partial V}\left(A_{f}[N] \cap \operatorname{im} v\right)-i_{\partial V}\left(A_{f}[N] \cap \nu_{f}^{-1} P\right) \stackrel{(3)}{=}[\partial \Delta]-[\xi P] .
\end{gathered}
$$

Here $P$ and $v$ are defined in [CS16, Proof of Lemma 4.8]. Equality (3) follows because

- $A_{f}[N] \cap \nu_{f}^{-1} P=[\xi P]$ by [CS16, Lemma 3.2.a $]$.

- $A_{f}[N] \cap \operatorname{im} v=\left[v\left(1_{2} \times S^{3}\right)\right]=[\partial \Delta]$ since

$$
\left(A_{f}[N] \cap \operatorname{im} v\right) \cap_{\operatorname{im} v}\left[v\left(S^{2} \times 1_{3}\right)\right]=A_{f}[N] \cap_{C_{f}} v\left(S^{2} \times 1_{3}\right) \stackrel{(2)}{=} A_{f}[N] \cap_{C_{f}} S_{f}^{2}=1 .
$$

Here equality (2) holds because $v\left(S^{2} \times 1_{3}\right)$ is homologous to $S_{f}^{2}$ in $C_{f}$.

Lemma 5.2. For every $y \in K_{u, l}$ there is a class $\widehat{z^{2}} \in H_{4}\left(W ; \mathbb{Z}_{d}\right)$ such that

(a) $\overline{z^{2}}:=\widehat{z^{2}}+n[t] \in j_{W}^{-1} \rho_{d} z^{2} \subset H_{4}\left(W, \partial ; \mathbb{Z}_{d}\right)$ for some $n \in \mathbb{Z}_{d}$;

(b) $[t]^{2}=\left(\widehat{z^{2}}\right)^{2}=0 \in \mathbb{Z}_{d}$ and $[t] \cap_{W} \widehat{z^{2}}=2 \in \mathbb{Z}_{d}$.

The proof is given later in this section. ${ }^{3}$

Proof of Lemma 2.1.a. The lemma follows by [CS16, Lemma 4.8] and Lemmas 5.1.b, 5.2. Indeed,

$$
\overline{z^{2}} \cap_{W}\left(z^{2}-p_{W}^{*}\right)=\overline{z^{2}} \cap_{W} \overline{z^{2}}-\overline{z^{2}} \cap_{W} p_{W}^{*} \stackrel{(2)}{=}\left(\widehat{z^{2}}+n[t]\right)^{2}-\left(\widehat{z^{2}}+n[t]\right) \cap_{W} 2 m[t] \stackrel{(3)}{=} 4 n-4 m .
$$

Here

- equality (2) holds by Lemma 5.1.b and property (a) of Lemma 5.2,

- equality (3) holds by property (b) of Lemma 5.2.

In the proof of Lemma 2.1.c we will use not only the statement of Lemma 5.2 but also the following definition, which is also used in the proof of Lemma 5.2.

Definition of $a, s, \widehat{z^{2}}$ for $y \in K_{u, l}$. By Lemma 5.1.a there is a representative

$$
a \in C_{4}(V) \text { of } Z \cap V \in H_{4}(V, \partial) \text { such that } \partial a=\partial \Delta-\xi P .
$$

(Such a representative is obtained from a representative $a^{\prime} \in C_{4}(V)$ of $Z \cap V \in H_{4}(V, \partial)$ such that $\partial a^{\prime}=\partial \Delta-\xi P+\partial a^{\prime \prime}$ for some $a^{\prime \prime} \in C_{4}(\partial V)$ by the formula $a:=a^{\prime}-a^{\prime \prime}$.)

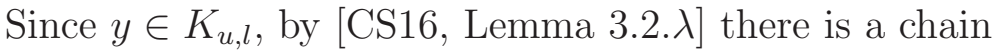

$$
s \in C_{4}\left(C_{f} \times 0 ; \mathbb{Z}_{d}\right) \text { such that } \partial s=2 \xi P \times 0 .
$$

Define

$$
\widehat{z^{2}}:=\left[2 a-2 \Delta-2 \xi P \times\left[0, \frac{1}{2}\right]+s\right] \in H_{4}\left(W ; \mathbb{Z}_{d}\right) .
$$

\footnotetext{
${ }^{3}$ Equality (3) from [CS16, Proof of Lemma 4.3.a] also holds by [CS16, Proof of Lemma 4.7] and by Lemma 5.2.a for $d=2$ because $\eta^{\prime}\left(\operatorname{id} \partial C_{f_{0}}, Y_{f_{0}, y}\right)=\overline{z^{2}} \cap_{W} z^{2}=\overline{z^{2}} \cap_{W} \overline{z^{2}}=2[t] \cap_{W} \widehat{z^{2}}=0 \in \mathbb{Z}_{2}$.
} 
Proof of Lemma 5.2. We have

$\rho_{d} z^{2} \cap W_{-} \stackrel{(1)}{=} 2 \rho_{d} i_{V, W_{-}}(Z \cap V)=[2 a]_{W_{-}, \partial}=\left[2 a-2 \xi P \times\left[0, \frac{1}{2}\right]+s\right]_{W_{-}, \partial}=\widehat{z^{2}} \cap W_{-}=j_{W} \widehat{z^{2}} \cap W_{-}$,

where equality (1) follows by Lemma 4.1. Hence by the cohomology exact sequence of the pair $\left(W, W_{-}\right)$(cf. diagram $\left.{ }^{*}\right)$ in [CS16, Proof of Lemma 4.8]) $\rho_{d} z^{2}=j_{W}\left(\widehat{z^{2}}+n[t]\right)$ for some $n \in \mathbb{Z}_{d}$. Thus property (a) holds.

Let us prove property (b). We have $[t]^{2}=\left[S^{2} \times 0 \times S^{2}\right] \cap_{S^{2} \times D^{3} \times S^{2}}\left[S^{2} \times 1_{3} \times S^{2}\right]=0$. Since the support of $\widehat{z^{2}}$ is in $W^{\prime} \cup \partial C_{f} \times\left[0, \frac{1}{2}\right] \cup C_{f} \times 0$ and this space is the boundary of a connected component of $W-W^{\prime}$, we have $\left(\widehat{z^{2}}\right)^{2}=0$. Also

$$
[t] \cap_{W} \widehat{z^{2}}=[t] \cap_{W_{-}}\left(\widehat{z^{2}} \cap W_{-}\right)=[t] \cap_{W_{-}}[2 a]_{W_{-}, \partial}=2[t] \cap_{\partial W_{-}}[\partial a]=2[t] \cap_{t \times \partial \Delta}[\partial \Delta]=2 .
$$

Here the homology classes are taken in the space indicated under ' ' (so $[t]$ has different meaning in different parts of the formula), and $\widehat{z^{2}} \cap W_{-}=[2 a]_{W_{-}, \partial}$ is proved in the proof of (a).

Proof of Lemma 2.1.c. Take any bundle isomorphism $\varphi: \partial C_{0} \rightarrow \partial C_{1}$ given by [CS16, Lemma 2.5]. Take a closed oriented 3-submanifold $P \subset N$ realizing $y \in H_{f_{0}}=H_{f_{1}}$. For $k=0,1$ construct the maps $v_{j k}, j=0,1,2,3$, manifolds $V_{k} \subset C_{k}, \widehat{V}_{k}, W_{k}^{\prime}$ and $W_{k}$, chains $a_{k}, s_{k}$ and classes $Z_{k}, z_{k}, \widehat{z_{k}^{2}}$ as in [CS16, Proof of Lemma 4.8] and above. (So unlike in other parts of this paper, subscript 0 of a manifold does not mean deletion of a codimension 0 ball from the manifold.) Define

$$
W:=W_{0} \cup_{\varphi \times \text { id } I: \partial C_{0} \times I \rightarrow \partial C_{1} \times C S 16} W_{1} .
$$

The manifold $W$ just defined should not be confused with the manifolds which were previously denoted $W$ but are now denoted $W_{0}$ and $W_{1}$. The same remark holds for $z, Z, \widehat{V}$ constructed below.

Consider the following segment of the ('cohomological') Mayer-Vietoris sequence:

$$
H_{6}(W, \partial) \stackrel{r_{W_{0}} \oplus r_{W_{1}}}{\longrightarrow} H_{6}\left(W_{0}, \partial\right) \oplus H_{6}\left(W_{1}, \partial\right) \stackrel{r_{0} \oplus\left(-r_{1}\right)}{\longrightarrow} H_{4}\left(\partial C_{0}\right) .
$$

Here $r_{k}$ is the composition $H_{6}\left(W_{k}, \partial\right) \stackrel{\partial}{\longrightarrow} H_{5}\left(\partial W_{k}\right) \stackrel{r_{\partial C_{0}}}{\longrightarrow} H_{4}\left(\partial C_{0}\right)$. We have

$$
r_{k} Z_{k}=\left(\partial Z_{k}\right) \cap \partial C_{0}=Y_{f_{k}} \cap \partial C_{0}=\partial\left(Y_{f_{k}} \cap C_{k}\right) \stackrel{(4)}{=} \partial A_{k}[N] \stackrel{(5)}{=} \partial A_{1-k}[N] \stackrel{(6)}{=} r_{1-k} Z_{1-k} \in H_{4}\left(\partial C_{0}\right) \text {. }
$$

Here

- equality (4) holds by descriptions of of joint Seifert classes [CS16, Lemma 3.13.a];

- equality (5) holds by agreement of Seifert classes [CS16, Lemma 3.5.a];

- equality (6) holds analogously to the previous set of equalities.

Hence there exists $Z \in H_{6}(W, \partial)$ such that $Z \cap W_{k}=Z_{k}$. Denote

$$
\widehat{V}:=\widehat{V}_{0} \bigcup_{\varphi: \nu_{0}^{-1} P \rightarrow \nu_{1}^{-1} P} \widehat{V}_{1} \subset W^{\prime} \quad \text { and } \quad z:=Z+[\widehat{V}] \in H_{6}(W, \partial) .
$$

Clearly, $z \cap W_{k}=z_{k} \cdot{ }^{4}$

Take $\widehat{z_{k}^{2}} \in H_{4}\left(W ; \mathbb{Z}_{d}\right)$ given by Lemma 5.2. Then by Lemmas 5.1.b and 5.2 $\overline{z_{k}^{2}} \cap_{W} p_{W}^{*}=4 m_{k}=\widehat{z_{k}^{2}} \cap_{W} p_{W}^{*} \quad$ and $\quad \overline{z_{k}^{2}} \cap_{W} z_{k}^{2}=\overline{z_{k}^{2}} \cap_{W} \overline{z_{k}^{2}}=4 n_{k}=2 \widehat{z_{k}^{2}} \cap_{W} \overline{z_{k}^{2}}=2 \widehat{z_{k}^{2}} \cap_{W} z_{k}^{2}$.

\footnotetext{
${ }^{4}$ Note that it is not assumed either that $(W, z)$ is a spin null-bordism of anything or that $\rho_{d} \partial z^{2}=0$.
} 
Hence

$$
\eta\left(f_{k}, y\right)=\rho_{\widehat{d}}\left(\widehat{z_{k}^{2}} \cap_{W_{k}}\left(2 z_{k}^{2}-p_{W_{k}}^{*}\right)\right)=\rho_{\widehat{d}}\left(\widehat{z_{k}^{2}} \cap_{W}\left(2 z^{2}-p_{W}^{*}\right)\right) .
$$

Take a weakly unlinked section $\xi_{0}: N_{0} \rightarrow \partial C_{0}$ of $f_{0}$. By [CS16, Lemma 3.4] $\xi_{1}:=\varphi \xi_{0}$ is an unlinked section of $f_{1}$. Hence

$$
\partial a_{1}-\partial \Delta_{1}=-\xi_{1} P=-\xi_{0} P=\partial a_{0}-\partial \Delta_{0} \quad \text { and } \quad \partial s_{1}=2 \xi_{1} P=2 \xi_{0} P=\partial s_{0} .
$$

Identify $M_{\varphi}$ with $M_{\varphi} \times 0 \subset \partial W$ and subsets of $M_{\varphi}$ with the corresponding subsets of $W$. Denote

$$
\widehat{a}:=\left[\Delta_{0}-a_{0}+a_{1}-\Delta_{1}\right] \in H_{4}\left(\widehat{V} ; \mathbb{Z}_{d}\right) \quad \text { and } \quad s:=\left[s_{0}-s_{1}\right] \in H_{4}\left(M_{\varphi} ; \mathbb{Z}_{d}\right)
$$

Then by the definition of $\widehat{z_{k}^{2}}$

$$
\widehat{z_{0}^{2}}-\widehat{z_{1}^{2}}=i_{\varphi} s-2 i \widehat{a}, \quad \text { where } \quad i_{\varphi}:=i_{M_{\varphi}, W} \quad \text { and } \quad i:=i_{\widehat{V}, W} .
$$

We have $i_{\varphi} s \cap_{W} p_{W}^{*}=s \cap_{M_{\varphi}} p_{M_{\varphi}}^{*}=0$.

Since

$$
\left(z \cap M_{\varphi}\right) \cap_{M_{\varphi}} S_{f_{0}}^{2}=\left(\partial z_{0} \cap C_{0}\right) \cap_{C_{0}} S_{f_{0}}^{2}=Y_{f_{0}} \cap_{C_{0}} S_{f_{0}}^{2}=1,
$$

$z \cap M_{\varphi}$ is a joint Seifert class for $\varphi$. Then

$$
i_{\varphi} s \cap_{W} z^{2}=\left(s \cap \partial C_{0}\right) \cap_{\partial C_{0}}\left(z^{2} \cap \partial C_{0}\right) \stackrel{(2)}{=} 2 \xi_{0} y \cap_{\partial C_{0}} \nu_{0}^{!} \beta=2 \beta \cap_{N} y
$$

where

- $\beta \in H_{1}\left(N ; \mathbb{Z}_{d}\right)$ is a lifting of $\beta\left(f_{0}, f_{1}\right)$;

- equality (2) follows because we have $s \cap \partial C_{0}=2\left[\xi_{0} P\right]=2 \xi_{0} y$ and because we have the identity $z^{2} \cap \partial C_{0}=\left(z \cap M_{\varphi}\right)^{2} \cap \partial C_{0}=\nu_{0}^{!} \beta$ by the definition of $\beta\left(f_{0}, f_{1}\right)$.

We have

$$
\begin{gathered}
z^{2} \cap_{W} i \widehat{a} \stackrel{(1)}{=}(Z+[\widehat{V}])^{2} \cap_{W} i \widehat{a} \stackrel{(2)}{=} Z^{2} \cap_{W} i \widehat{a}+2 Z \cap_{W}[\widehat{V}] \cap_{W} i \widehat{a} \stackrel{(3)}{=} \\
=(Z \cap \widehat{V})_{\widehat{V}}^{2} \cap_{\widehat{V}} \widehat{a}+2 i(Z \cap \widehat{V}) \cap_{W} i \widehat{a} \stackrel{(4)}{=}(\widehat{a})_{\widehat{V}}^{3}+2(i \widehat{a})^{2} \stackrel{(5)}{=}(\widehat{a})_{\widehat{V}}^{3}
\end{gathered}
$$

where

- equality (1) follows by the definition of $z$;

- equalities (2) and (5) follow because $\widehat{V} \subset W^{\prime}$, so $[\widehat{V}]^{2}=0$ and $(i \widehat{a})^{2}=0$;

- equality (3) is obvious;

- equality (4) follows because $Z \cap \widehat{V}=\widehat{a}$ by the definition of $a_{0}, a_{1}, \widehat{a}$.

Therefore $i \widehat{a} \cap_{W}\left(2 z^{2}-p_{W}^{*}\right)=2(\widehat{a})_{\widehat{V}}^{3}-\widehat{a} \cap_{\widehat{V}} p_{\widehat{V}}^{*} \equiv \overline{\overline{12}} 0$ by [Wa66, Theorem 5].

Now the lemma follows because

$$
\left(\widehat{z_{0}^{2}}-\widehat{z_{1}^{2}}\right) \cap_{W}\left(2 z^{2}-p_{W}^{*}\right)=2 i_{\varphi} s \cap_{W} z^{2}-i_{\varphi} s \cap_{W} p_{W}^{*}-2 i \widehat{a} \cap_{W}\left(2 z^{2}-p_{W}^{*}\right) \underset{\overline{24}}{\equiv} 4 \beta \cap_{N} y .
$$




\section{$6 \quad$ Idea of the proof of Lemma 2.1}

Here we present Lemma 6.1 which we include for expositional purposes. For $N=S^{1} \times S^{3}$, this lemma introduces the constructions used in the proof of Lemma 2.1. It can also be used to simplify the proof of Theorem 1.2. However, we do not present this simplification here. Hence Lemma 6.1 is not used in the remainder of the paper.

The standard embedding $\tau_{0}: S^{1} \times S^{3} \rightarrow S^{7}$ is defined in [CS16, §2.4].

Lemma 6.1. $\eta\left(\tau_{0}, y\right)=0$ for every $y \in H_{3}\left(S^{1} \times S^{3}\right)$.

Proof. Define an extension

$$
\mathrm{i}: D^{2} \times D^{4} \rightarrow S^{7} \quad \text { of } \tau_{0} \text { by } \quad \mathrm{i}(x, y):=\left(y \sqrt{2-|x|^{2}}, 0,0, x\right) / \sqrt{2} \text {. }
$$

Take an embedding $v_{0}: S^{5} \rightarrow S^{7}-\mathrm{i}\left(S^{1} \times D^{4}\right)$ whose linking coefficient with $\mathrm{i}\left(S^{1} \times D^{0}\right)$ is equal to $y \cap_{S^{1} \times S^{3}}\left[S^{1} \times 1_{3}\right]$. We omit the subscript $\tau_{0}$ in this proof. As $\operatorname{lk}\left(\widehat{A} y, \tau_{0}\left(S^{1} \times 1_{3}\right)\right)=y \cap_{S^{1} \times S^{3}}\left[S^{1} \times 1_{3}\right]$, we have $\widehat{A} y=\left[v_{0}\left(S^{5}\right)\right] \in H_{5}(C) \cong \mathbb{Z}$. We also have $\widehat{A} y=i_{C} \nu^{!} y$. Take a representative $P$ of $y$ and a chain

$$
V \in C_{6}(C) \text { such that } \partial V=\nu^{-1} P-v_{0}\left(S^{5}\right) .
$$

Since $C$ is parallelizable, $v_{0}$ extends to an embedding $v_{2}: S^{5} \times D^{2} \rightarrow \operatorname{Int} C=\operatorname{Int} C \times \frac{1}{2}$ which is orientation-preserving and transversal to $V$ and such that im $v_{2} \cap V=v_{0}\left(S^{5}\right)$. Extend $v_{2}$ to an orientation-preserving embedding $v_{3}: S^{5} \times D^{3} \rightarrow \operatorname{Int}(C \times I)$. Let

$$
W_{-}:=C \times I-\operatorname{Intim} v_{3} \text { and } W:=W_{-} \cup_{\left.v_{3}\right|_{S^{5} \times S^{2}}} D^{6} \times S^{2} .
$$

Consider the cohomology exact sequence of the pair $\left(W, W_{-}\right)$in the following Poincaré dual form (analogous to the sequence $\left(^{*}\right)$ in [CS16, Proof of Lemma 4.8]):

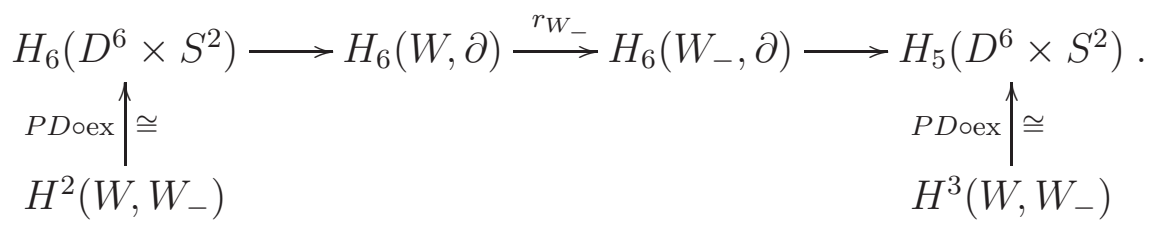

Since $H_{5}\left(D^{6} \times S^{2}\right)=0$, the map $r_{W_{-}}$is an epimorphism. Take any

$$
Z \in r_{W_{-}}^{-1}\left(A[N] \times I \cap W_{-}\right) \subset H_{6}(W, \partial) .
$$

Denote

$$
\widehat{V}:=V \cup D^{6} \times 1_{2} \quad \text { and } \quad z:=Z+[\widehat{V}] \in H_{6}(W, \partial) .
$$

Since $H_{5}\left(D^{6} \times S^{2}\right)=0$, the spin structure on $W_{-}$coming from $S^{7} \times I$ extends to $W$. Clearly, $\partial W \underset{\text { spin }}{=} \partial(C \times I) \underset{\text { spin }}{=} M$ (for the 'boundary' spin structure on $M$ coming from $C \times I)$. Since

$$
\partial_{W} Z=\partial_{C \times I}(A[N] \times I)=Y_{0} \quad \text { and } \quad \partial_{W}[\widehat{V}]=\left[\nu^{-1} P \times \frac{1}{2}\right]=i_{M} \widehat{A} y, \quad \text { we have } \quad \partial_{W} z=Y_{y} .
$$

By [CS16, Lemma 4.7] $\partial_{W} z^{2}=Y_{y}^{2}=0$. So $z^{2} \in \operatorname{im} j_{W}$. Analogously to $(*)$ we obtain an isomorphism $H_{4}(C \times I) \cong H_{4}(W)$ commuting with $i_{C \times I}: H_{4}(M) \rightarrow H_{4}(C \times I)$ and $i_{W}: H_{4}(M) \rightarrow$ $H_{4}(W)$. Since $i_{C \times I}$ is onto, $i_{W}$ is onto. Hence $j_{W}=0$. Thus $z^{2}=0$. So we take $\overline{z^{2}}:=0$ and obtain that $\eta\left(\tau_{0}, y\right)=\overline{z^{2}} \cap_{W}\left(z^{2}-p_{W}^{*}\right)=0 . .^{5}$

\footnotetext{
${ }^{5}$ We essentially proved that if $\widehat{A}_{f} y$ is spherical, then $\eta(f, y)=0$.
} 


\section{$7 \quad$ Discussion of the action of knots}

Remark 7.1 (The action of knots in Theorem 1.6). (a) Take any $[f] \in E^{7}(N)$. Let

$$
O(f)=O([f]):=\left\{[f \# g]:[g] \in E^{7}\left(S^{4}\right)\right\}
$$

be the orbit of $[f]$ under the action of $E^{7}\left(S^{4}\right)$. We have $O(f)=\beta_{u, l}^{-1}(b)$ when $[f] \in \beta_{u, l}^{-1}(b)$ by [CS16, Theorem 1.2] and the additivity of $\varkappa, \lambda$ and $\beta$ [CS16, Lemmas 2.3 and 2.9].

Define the inertia group of $f, I(f) \subset E^{7}\left(S^{4}\right)=\mathbb{Z}_{12},{ }^{6}$ to be the subgroup of isotopy classes in $E^{7}\left(S^{4}\right)$ which do not change $[f]$ after embedded connected sum:

$$
I(f)=I([f]):=\left\{[g] \in E^{7}\left(S^{4}\right):[f \# g]=[f]\right\}
$$

For some cases this orbit and group are found in terms of $u, l, b$ in Corollaries 1.5 and 1.8.

(b) The indeterminancy in the classification of Theorem 1.6.c corresponds to the fact that we do not always know $\operatorname{im} \theta_{u, l, b}$. Thus determining $\operatorname{im} \theta_{u, l, b}$ becomes a key problem. This image is found in this paper when either $u=0$ or $2 \rho_{d} \bar{l}=0$ (Corollary 1.8) or in the cases $(1,2,3)$ of Remark 3.1.

For general $u, l$ and simple enough $N$ there are some $u, l$ such that for each $b$ the methods of this paper do not completely determine $\operatorname{im} \theta_{u, l, b}$. E.g. let $N=\left(S^{1} \times S^{3}\right) \#\left(S^{2} \times S^{2}\right)$. Then $x \cap_{N} y \cap_{N} u=0$ for each $x, y \in H_{3}$ and $u \in H_{2}$. Take the standard bases for $H_{2} \cong H_{2}\left(S^{2} \times S^{2}\right)$ and for $H_{3} \cong H_{3}\left(S^{1} \times S^{3}\right)$. The pair $((0,6), l)$ is symmetric, where $l(x, y):=x y$. So by Theorem 1.6.a there is an embedding $f: N \rightarrow S^{7}$ such that $(\varkappa \times \lambda)(f)=((0,6), l)$. Then $d:=\operatorname{div}(0,6)=6$, $\widehat{d}=6$ and $\bar{l}: H_{3} \rightarrow H_{1}$ is 'the identity'. Hence $\rho_{d} \bar{l}$ is surjective. Thus $C_{u, l}=\frac{H_{1} \otimes \mathbb{Z}_{d}}{2 H_{1} \otimes \mathbb{Z}_{d}} \cong \mathbb{Z}_{2}$, $K_{u, l}=3 H_{3} \cong \mathbb{Z}$ and the pairing $\cap_{d}: C_{u, l} \times K_{u, l} \rightarrow \mathbb{Z}_{d}$ is given by $\rho_{2} z \cap_{d} 3 y=3 \rho_{d}\left(z \cap_{N} y\right)$ for each $z \in H_{1}$ and $y \in H_{3}$. So this pairing is trivial mod3. Then by Theorem 1.6.c $\theta_{u, l, b}=\theta_{u, l, b^{\prime}}$ for each $b, b^{\prime} \in C_{u, l}$. Both the trivial and the non-trivial homomorphisms $K_{u, l} \rightarrow 4 \mathbb{Z}_{6} \cong \mathbb{Z}_{3}$ fit into the conclusion of Theorem 1.6.c, so Theorem 1.6.c does not allow us to completely determine $\operatorname{im} \theta_{u, l, b}$.

Problem 7.2. (a) Characterize those closed connected orientable 4-manifold with torsion free $H_{1}$ such that for every embeddings $f: N \rightarrow S^{7}$ and $g: S^{4} \rightarrow S^{7}$ the embeddings $f \# g$ and $f$ are isotopic (in other words, the action \# of $E^{7}\left(S^{4}\right)$ on $E^{7}(N)$ is trivial).

Cf. Corollary 1.4. If $H_{1}=0$, then this property is equivalent to $H_{2}^{D I F F}$ containing no elements divisible either by 4 or by 3. E.g. $N=\mathbb{C} P^{2}$ satisfies this property (because $\sigma\left(\mathbb{C} P^{2}\right)=1$ ).

(b) Characterize those $f$ for which $|O(f)|=12$ (i.e. $|I(f)|=1$ ), and those $f$ for which $|O(f)|=1$ (i.e. $|I(f)|=12)$.

Addendum 7.3. For every $l \in \mathbb{Z}-\{0\}$ there is a map $\psi_{l}: \mathbb{Z} \times \mathbb{Z}_{2 l} \rightarrow \mathbb{Z}_{12}$ such that for every $a, a^{\prime} \in \mathbb{Z}_{12}$ and $l, b, l^{\prime}, b^{\prime} \in \mathbb{Z}$ we have $a \# \tau(l, b)=a^{\prime} \# \tau\left(l^{\prime}, b^{\prime}\right)$ if and only if

$$
\begin{gathered}
\text { either } \quad l=l^{\prime}=0, \quad b=b^{\prime} \quad \text { and } a \equiv a^{\prime} \bmod 2 \operatorname{gcd}(b, 6), \\
\text { or } \quad l=l^{\prime} \neq 0, \quad b \equiv b^{\prime} \bmod 2 l \quad \text { and } \quad \rho_{12}\left(a-a^{\prime}\right)=\psi_{l}\left([b / 2 l], \rho_{2 l} b\right)-\psi_{l}\left(\left[b^{\prime} / 2 l\right], \rho_{2 l} b\right) \text {. }
\end{gathered}
$$

Proof. By Theorem 1.2.b if either $l \neq l^{\prime}$ or $b \not \equiv b^{\prime} \bmod 2 l$, then the equivalence is clear because neither of the two assertions holds.

Assume that $l=l^{\prime}$ and $b \equiv b^{\prime} \bmod 2 l$. Let $\tau:=\tau(l, b)$ and $\tau^{\prime}=\tau\left(l, b^{\prime}\right)$. By the Isotopy Classification Theorem 2.5 and Theorem 1.2.b $a \# \tau=a^{\prime} \# \tau^{\prime} \Leftrightarrow \eta\left(a \# \tau, a^{\prime} \# \tau^{\prime}\right)=0$. Since $\operatorname{div} \varkappa(\tau)=0$, we may use Corollary 1.8.b.

\footnotetext{
${ }^{6}$ The inertia group of $f$ is just the stabilizer of $[f]$ under the action of $E^{7}\left(S^{4}\right)$. We use the word 'inertia' following its use for the action of the group homotopy spheres on the diffeomorphism classes of smooth manifolds: see the last paragraph of Remark 1.1.
} 
If $l=0$, then $b=b^{\prime}$. By Theorem 1.2.b and Corollary 1.8.b im $\theta_{0,0, b}$ consists of elements of $\mathbb{Z}_{24}$ which are divisible by $4 \operatorname{gcd}(b, 6)$. Hence by Addendum 1.7 and the transitivity of $\eta$ (Lemma 2.4)

$$
\eta\left(a \# \tau, a^{\prime} \# \tau^{\prime}\right)=\rho_{4 \operatorname{gcd}(b, 6)}\left(2 a-2 a^{\prime}\right) \in \mathbb{Z}_{4 \operatorname{gcd}(b, 6)} .
$$

If $l \neq 0$, then by Theorem 1.2.b and Corollary 1.8.b $\operatorname{im} \theta_{0, l, b}=0$ and $\eta\left(a \# \tau, a^{\prime} \# \tau^{\prime}\right) \in 2 \mathbb{Z}_{24}$. Identify $\mathbb{Z}_{2 l}$ and $\{0,1, \ldots, 2 l-1\}$. For every $x \in \mathbb{Z}_{2 l}$ and $k \in \mathbb{Z}$ define

$$
\psi_{l}(k, x):=\frac{1}{2} \eta(\tau(l, x), \tau(l, x+2 k l)) \in \mathbb{Z}_{12} .
$$

Define $\bar{\tau}:=\tau\left(l, \rho_{2 l} b\right)$. Then by Addendum 1.7 and the transitivity of $\eta$ (Lemma 2.4)

$\frac{\eta\left(a \# \tau, a^{\prime} \# \tau^{\prime}\right)}{2}=\rho_{12}\left(a-a^{\prime}\right)-\frac{\eta(\bar{\tau}, \tau)}{2}+\frac{\eta\left(\bar{\tau}, \tau^{\prime}\right)}{2}=\rho_{12}\left(a-a^{\prime}\right)-\psi_{l}\left([b / 2 l], \rho_{2 l} b\right)+\psi_{l}\left(\left[b^{\prime} / 2 l\right], \rho_{2 l} b\right)$.

The two formulas for $\eta\left(a \# \tau, a^{\prime} \# \tau^{\prime}\right)$ above imply the stated equivalence.

Remark 7.4 (An approach to the action of knots). Let us explain the ideas required to move from the classification modulo knots in [CS16] to the main results of this paper. We briefly recall and continue the discussion in [CS16, 1.4].

Suppose that $f_{0}, f_{1}: N \rightarrow S^{7}$ are embeddings. Assume that $f_{1}$ is isotopic to $f_{0} \# g$ for some embedding $g: S^{4} \rightarrow S^{7}$. By [CS16, Isotopy Classification Modulo Knots Theorem 2.8] this is equivalent to $\lambda\left(f_{0}\right)=\lambda\left(f_{1}\right), \varkappa\left(f_{0}\right)=\varkappa\left(f_{1}\right)$ and $\beta\left(f_{0}, f_{1}\right)=0$. The complements $C_{0}$ and $C_{1}$ may be glued together along a bundle isomorphism $\varphi: \partial C_{0} \rightarrow \partial C_{1}$ to form a parallelizable closed 7-manifold $M=C_{0} \cup_{\varphi}\left(-C_{1}\right)$. Recall that $d:=\operatorname{div} \varkappa\left(f_{0}\right)$ is the divisibility of $\varkappa\left(f_{0}\right) \in H_{2}$. By the assumption on $f_{0}, f_{1}$ there is a joint Seifert class $Y \in H_{5}(M)$ such that $\rho_{d} Y^{2}=0$, i.e. a d-class [CS16, Lemma 4.1]. There is a spin null-bordism $(W, z)$ of $\left(M_{\varphi}, Y\right)$, since $\Omega_{7}^{\text {Spin }}\left(\mathbb{C} P^{\infty}\right)=0$. Since $\rho_{d} Y^{2}=0$, the class $\rho_{d} z^{2} \in H_{4}\left(W, \partial ; \mathbb{Z}_{d}\right)$ lifts to $\overline{z^{2}} \in H_{4}\left(W ; \mathbb{Z}_{d}\right)$. Recall that $p_{W}^{*} \in H_{4}(W, \partial)$ is the Poincaré dual of $p_{W}$, the spin Pontrjagin class of $W$. We then verified that the Kreck invariant,

$$
\eta(\varphi, Y):=\overline{z^{2}} \cap_{W} \rho_{\widehat{d}}\left(z^{2}-p_{W}^{*}\right) \in \mathbb{Z}_{\widehat{d}}
$$

determines the surgery obstruction for $W$ to be spin diffeomorphic to the product $C_{0} \times I$ [CS16]. We proved that $\eta(\varphi, Y)$ is independent of the choices of $W, z, \overline{z^{2}}$ for a fixed bundle isomorphism $\varphi$ and $d$-class $Y$ [CS16, §4.1]. We also proved that $\eta(\varphi, Y)$ is independent of the choice of $\varphi$ : for the precise statement, see [CS16, Lemma 4.3.c]. So we need to know the various values of $\eta(\varphi, Y)$ arising from the different possible choices of $Y$. These choices are described in [CS16, Description of $d$-classes Lemma 4.7]. The achievement of this paper is showing that the change of $\eta(\varphi, Y)$ under a change of $Y$ is precisely determined by $\theta_{u, l, b}$, and proving the properties of $\theta_{u, l, b}($ Lemma 2.1).

It is well known that there are pairs of 4-manifolds $N_{0}$ and $N_{1}$ which are homeomorphic but not diffeomorphic (see e.g. [GS99]). It is natural to ask about the relationship between $E^{7}\left(N_{0}\right)$ and $E^{7}\left(N_{1}\right)$ in this case.

Conjecture 7.5. Let $N_{0}$ and $N_{1}$ be closed connected 4-manifolds.

(a) A homeomorphism $h: N_{0} \rightarrow N_{1}$ gives rise to a geometrically defined bijection $E_{h}: E^{7}\left(N_{0}\right) \rightarrow E^{7}\left(N_{1}\right)$.

(b) A smooth $h$-cobordism $\left(W ; N_{0}, N_{1}\right)$ gives rise to a geometrically defined bijection $E_{\left(W ; N_{0}, N_{1}\right)}: E^{7}\left(N_{0}\right) \rightarrow E^{7}\left(N_{1}\right)$. 


\section{References}

[BH70] J. Boechat and A. Haefliger, Plongements differentiables de varietes de dimension 4 dans $\mathbb{R}^{7}$, Essays on topology and related topics, Springer, 1970, 156-166.

[CRS07] M. Cencelj, D. Repovš and M. Skopenkov, Homotopy type of the complement of an immersion and classification of embeddings of tori, Uspekhi Mat. Nauk, 62:5 (2007) 165166; English transl: Russian Math. Surveys, 62:5 (2007)

[CS11] D. Crowley and A. Skopenkov. A classification of smooth embeddings of 4-manifolds in 7-space, II, Intern. J. Math., 22:6 (2011) 731-757, arxiv:math/0808.1795.

[CRS11] M. Cencelj, D. Repovš and M. Skopenkov, Classification of knotted tori in the 2metastable dimension, Mat. Sbornik, 203:11 (2012), 1654-1681. arxiv:math/0811.2745.

[CS16] D. Crowley and A. Skopenkov, Embeddings of non-simply-connected 4-manifolds in 7space. I. Classification modulo knots, Moscow Math. J., to appear. arXiv:1611.04738.

[Do87] S. K. Donaldson, The orientation of Yang-Mills moduli spaces and 4-manifold topology, J. Differential Geom. 26 (1987), 397-428.

[GS99] * R. Gompf and A. Stipsicz, 4-Manifolds and Kirby Calculus, 1999, Amer. Math. Soc., Providence, RI.

[KS91] M. Kreck and S. Stolz, Some nondiffeomorphic homeomorphic homogeneous 7-manifolds with positive sectional curvature, J. Diff. Geom. 33 (1991) 465-486.

[Le70] J. Levine, Inertia groups of manifolds and diffeomorphisms of spheres, Amer. J. Math. 92 (1970) 243-258.

[Sc73] R. Schultz, Homotopy decompositions of equivariant function spaces. I, Math. Z. 131 (1973), 49-75.

[Sk08] * A. Skopenkov. Embedding and knotting of manifolds in Euclidean spaces, London Math. Soc. Lect. Notes, 347 (2008) 248-342; arXiv:math/0604045.

[Sk08'] A. Skopenkov, Classification of smooth embeddings of 3-manifolds in 6-space, Math. Zeitschrift, 260:3, 2008, 647-672, arxiv:math/0603429

[Sk10] A. Skopenkov, A classification of smooth embeddings of 4-manifolds in 7-space, I, Topol. Appl., 157 (2010) 2094-2110, arxiv:math/0808.1795.

[Sk11] M. Skopenkov. When is the set of embeddings finite? Intern. J. Math. $26: 7$ (2015), arxiv:1106.1878.

[Sk15] A. Skopenkov. Classification of knotted tori, Proc. A of the Royal Society of Edinburgh, to appear. arXiv: 1502.04470.

[Sk16c] * A. Skopenkov, Embeddings in Euclidean space: an introduction to their classification, to appear in Boll. Man. Atl. http://www.map.mpimbonn.mpg.de/Embeddings_in_Euclidean_space:_an_introduction_to_their_classification

[Sk16e] * A. Skopenkov, Embeddings just below the stable range: classification, to appear in Boll. Man. Atl. http://www.map.mpimbonn.mpg.de/Embeddings_just_below_the_stable_range:_classification 
[Sk16f] * A. Skopenkov, 4-manifolds in 7-space, to appear in Boll. Man. Atl. http://www.map.mpim-bonn.mpg.de/4-manifolds_in_7-space

[Sk16t] * A. Skopenkov, 3-manifolds in 6-space, to appear in Boll. Man. Atl. http://www.map.mpim-bonn.mpg.de/3-manifolds_in_6-space

[Vi73] O. Ya. Viro, Local knotting of submanifolds (in Russian) Mat. Sbornik, 90:2 (1973) 173183.

[Wa66] C. T. C. Wall, Classification Problems in Differential Topology. V. On Certain 6Manifolds, Invent. Math., 1 (1966) 355-374.

[Wi74] D. L. Wilkens, On the inertia group of certain manifolds, J. London Math. Soc. 9 $(1974 / 5), 537-548$.

In this list books, surveys and expository papers are marked by stars 